\title{
Miura-Reciprocal Transformation and Symmetries for the Spectral Problems of KdV and mKdV
}

\author{
Paz Albares (D) and Pilar Garcia Estévez *(D) \\ Departamento de Física Fundamental, Universidad de Salamanca, 37008 Salamanca, Spain; paz.albares@usal.es \\ * Correspondence: pilar@usal.es
}

check for updates

Citation: Albares, P.; Estévez, P.G. Miura-Reciprocal Transformation and Symmetries for the Spectral Problems of KdV and mKd. Mathematics 2021, 9 926. https://doi.org/10.3390/ math9090926

Academic Editor: Maria Luz Gandarias

Received: 22 March 2021

Accepted: 19 April 2021

Published: 22 April 2021

Publisher's Note: MDPI stays neutral with regard to jurisdictional claims in published maps and institutional affiliations.

Copyright: (c) 2021 by the authors. Licensee MDPI, Basel, Switzerland. This article is an open access article distributed under the terms and conditions of the Creative Commons Attribution (CC BY) license (https:// creativecommons.org/licenses/by/ $4.0 /)$.
Abstract: We present reciprocal transformations for the spectral problems of Korteveg de Vries (KdV) and modified Korteveg de Vries (mKdV) equations. The resulting equations, RKdV (reciprocal KdV) and $\mathrm{RmKdV}$ (reciprocal $\mathrm{mKdV}$ ), are connected through a transformation that combines both Miura and reciprocal transformations. Lax pairs for RKdV and RmKdV are straightforwardly obtained by means of the aforementioned reciprocal transformations. We have also identified the classical Lie symmetries for the Lax pairs of RKdV and RmKdV. Non-trivial similarity reductions are computed and they yield non-autonomous ordinary differential equations (ODEs), whose Lax pairs are obtained as a consequence of the reductions.

Keywords: symmetry groups; conservation laws; partial differential equations

\section{Introduction}

Hodograph transformations are transformations involving the interchange of dependent and independent variables [1,2]. When the variables are swapped, the space of independent variables is called the reciprocal space. Reciprocal transformations share this definition with hodograph transformations, but the former impose further requirements. Reciprocal transformations require the use of conservative forms together with the fulfillment of specific properties [3-6].

A very important advantage when dealing with reciprocal transformations is that many integrable equations happen to be related via reciprocal transformations. Two apparently unrelated equations [7] or even two complete hierarchies of partial differential equations (PDEs) [8,9] that are linked via reciprocal transformation, are versions of a unique problem. Reciprocal transformations were proven to be a useful instrument to transform equations with peakon solutions into equations that are integrable in the Painlevé sense $[6,10]$. For example, the composition of a Miura transformation [11,12] and a reciprocal transformation gives rise to the so called Miura-reciprocal transformations [9] that helps us relate two different hierarchies of differential equations.

A second significant advantage of reciprocal transformations is their utility in the identification of integrable PDEs which cannot be identified through algebraic tests such as the Painlevé test [13]. Our conjecture is that if an equation is integrable, there must be a transformation that turns the initial equation into a new one for which the Painlevé test is successful [6,8]. Similar ideas have been explored by Sakovich in [14-16].

A third bonus when using reciprocal transformations is their role in the derivation of Lax pairs. Although it is not always possible to find a Lax pair for a given equation, a reciprocal transformation can turn it into a different one whose Lax pair is known. Therefore, by undoing the reciprocal transformation in the Lax pair of the transformed equation, we can retrieve the Lax pair of the initial equation.

This paper is an explicit display of the previous statements when applied to the famous KdV and $\mathrm{mKdV}$ equations [17]. Nevertheless, we are dealing with the associated linear problems of these equations instead of the equations themselves. While the spectral problems for $\mathrm{KdV}$ and $\mathrm{mKdV}$ are very well known, the Lax pairs of their reciprocal versions 
are unknown. Furthermore, the Painlevé test cannot be applied to RKdV and RmKdV. Despite this, the reciprocal transformations can be extended to the eigenfunctions of the Lax pair, and the spectral problems for RKdV and RmKdV can be obtained, as described in Sections 2 and 3.

As $\mathrm{KdV}$ and $\mathrm{mKdV}$ are connected through a Miura transformation, we can conjecture that something similar should happen with their reciprocal versions. In section 4, we have successfully combined reciprocal transformations with the Miura transformations between $\mathrm{KdV}$ and $\mathrm{mKdV}$. The result is a reciprocal-Miura transformation between RKdV and RmKdV. Section 4 is devoted to this subject.

Finally, in Section 5, we shall calculate the classical Lie point symmetries for the spectral problems of RKdV and $R m K d V$, leading to a three parameter symmetry group in each case. We have identified some non-trivial reductions which yield the reduced ODE together with its associated spectral problem. The identification of the symmetries of the Lax pair implies the consideration of the spectral parameter as an independent variable according to ideas expressed by different authors [18-21].

\section{Reciprocal Transformations for $\mathrm{mKdV}$ and its Spectral Problem}

As it is well known [17], the celebrated $\mathrm{mKdV}$ equation

$$
u_{t}+u_{x x x}-6 u^{2} u_{x}=0
$$

has a two-component Lax pair which can be written in matrix form as

$$
\begin{aligned}
& \vec{\psi}_{x}=b \vec{\psi} \\
& \vec{\psi}_{t}=c \vec{\psi}
\end{aligned}
$$

where $\Lambda$ is the spectral parameter and $\vec{\psi}=\left(\psi_{1}, \psi_{2}\right)^{\top}$ is a two-component eigenfunction. Furthermore, $b$ and $c$ are the $2 \times 2$ matrices

$$
\begin{aligned}
& b=\left(\begin{array}{cc}
-u & \Lambda \\
\Lambda & u
\end{array}\right) \\
& c=\left(\begin{array}{cc}
u_{x x}-2 u^{3}+4 \Lambda^{2} u & 2 \Lambda\left[u^{2}-u_{x}-2 \Lambda^{2}\right] \\
2 \Lambda\left[u^{2}+u_{x}-2 \Lambda^{2}\right] & -u_{x x}+2 u^{3}-4 \Lambda^{2} u
\end{array}\right)
\end{aligned}
$$

Equation (1) can be written in conservative form as

$$
u_{t}=\left[2 u^{3}-u_{x x}\right]_{x}
$$

which allows us to introduce the following exact derivative

$$
d z=u d x+\left[2 u^{3}-u_{x x}\right] d t
$$

Therefore, a reciprocal transformation can be introduced as

$$
x=x(z, \tau)
$$

where

$$
\begin{aligned}
& d x=\frac{d z}{u}-\frac{2 u^{3}-u_{x x}}{u} d \tau \\
& t=\tau
\end{aligned}
$$


Equation (7) implies that the derivatives of $x(z, \tau)$ are

$$
\begin{aligned}
x_{z} & =\frac{1}{u} \\
x_{\tau} & =\frac{u_{x x}}{u}-2 u^{2}
\end{aligned}
$$

Hence, the reciprocal transformation (7), when applied to the derivatives, reads

$$
\begin{aligned}
& \frac{\partial}{\partial x}=u \frac{\partial}{\partial z} \\
& \frac{\partial}{\partial t}=\frac{\partial}{\partial \tau}+\left[2 u^{3}-u_{x x}\right] \frac{\partial}{\partial z}
\end{aligned}
$$

If we define

$$
u(x, t)=U(z, \tau)
$$

we have

$$
\begin{aligned}
& u_{x}=U U_{z} \\
& u_{x x}=U^{2} U_{z z}+U U_{z}^{2}
\end{aligned}
$$

and Equations (8) provide

$$
\begin{aligned}
& x_{z}=\frac{1}{U} \\
& x_{\tau}=U U_{z z}+U_{z}^{2}-2 U^{2}
\end{aligned}
$$

The cross derivatives of (12) yield

$$
\left[\frac{1}{U}\right]_{\tau}=\left[U U_{z z}+U_{z}^{2}-2 U^{2}\right]_{z}
$$

and finally

$$
U_{\tau}+\left[U^{3}\left(U_{z z}-U\right)\right]_{z}=0
$$

Equation (13) shall be denoted RmKdV in the future because it is the reciprocal version of $\mathrm{mKdV}$ (1) obtained through the reciprocal transformation (7) and (10).

\section{Spectral Problem of RmKdV}

An interesting remark is that Equation (13) should be integrable, due to its direct relation via a reciprocal transformation to an integrable one. Nevertheless, integrability tests such as those based in the Painlevé property [13] do not work for these equation because a leading index cannot be even identified. Similar problems appear for some well-known integrable equations such as Camassa-Holm [22] or Degasperis-Procesi [10]. In previous papers of one of the authors $[5,6,8]$, reciprocal transformations have been presented as an efficient method to identify the spectral problem of an equation without Painlevé property by means of its reciprocal relation to an equation which successfully passes the Painlevé test. This same procedure can be applied in order to get a Lax pair for Equation (13). Actually, we can introduce a transformed eigenvector defined as

$$
\vec{\Psi}(z, \tau)=\vec{\psi}(x, t)
$$

By using the transformation of the derivatives given in (9) we easily obtain the following Lax pair

$$
\begin{aligned}
& \vec{\Psi}_{z}=B \vec{\Psi} \\
& \vec{\Psi}_{\tau}=\Lambda C \vec{\Psi}
\end{aligned}
$$


where $B$ and $C$ are the $2 \times 2$ matrices

$$
\begin{aligned}
& B=\left(\begin{array}{cc}
-1 & \bar{U} \\
\Lambda & 1
\end{array}\right) \\
& C=\left(\begin{array}{cc}
4 \Lambda U & U U_{z z}+U_{z}^{2}-2 U U_{z}-4 \Lambda^{2} \\
U U_{z z}+U_{z}^{2}+2 U U_{z}-4 \Lambda^{2} & -4 \Lambda U
\end{array}\right)
\end{aligned}
$$

It is easy to check that the compatibility condition $\Psi_{z \tau}=\Psi_{\tau z}$ between the two components of (15) is precisely Equation (13).

\section{Reciprocal Transformations for KdV and Its Spectral Problem}

Let

$$
m_{t}+m_{x x x}-6 m m_{x}=0
$$

be the well-known Korteveg de Vries equation [17]. Equally renowned is the existence of the Miura transformation

$$
m_{1}=u^{2}-u_{x}, \quad m_{2}=u^{2}+u_{x}
$$

between two solutions $m_{1}, m_{2}$ of (17) and a solution $u$ of (1). Besides that, each of the components of the eigenvector $\vec{\psi}=\left(\psi_{1}, \psi_{2}\right)^{\top}$ of $\mathrm{mKdV}$ satisfies the KdV scalar Lax pair

$$
\begin{aligned}
& \left(\psi_{i}\right)_{x x}=\left(m_{i}-\lambda\right) \psi_{i} \\
& \left(\psi_{i}\right)_{t}=\left(2 m_{i}+4 \lambda\right)\left(\psi_{i}\right)_{x}-\left(m_{i}\right)_{x} \psi_{i}
\end{aligned}
$$

for $i=1,2$ and where

$$
\lambda=-\Lambda^{2}
$$

Equation (17) can be written in conservative form as

$$
m_{t}=\left[3 m^{2}-m_{x x}\right]_{x}
$$

The conservative Equation (20) allows us to introduce the exact derivative

$$
d y=m d x+\left[3 m^{2}-m_{x x}\right] d t
$$

that yields a reciprocal transformation

$$
x=x(y, T)
$$

where

$$
\begin{aligned}
& d x=\frac{d y}{m}-\frac{3 m^{2}-m_{x x}}{m} d T \\
& T=t
\end{aligned}
$$

The induced transformation of the derivatives is

$$
\begin{aligned}
& \frac{\partial}{\partial x}=m \frac{\partial}{\partial y} \\
& \frac{\partial}{\partial t}=\frac{\partial}{\partial T}+\left[3 m^{2}-m_{x x}\right] \frac{\partial}{\partial y}
\end{aligned}
$$

If we define

$$
m(x, t)=M(y, T)
$$


its derivatives are

$$
\begin{aligned}
& m_{x}=M M_{y} \\
& m_{x x}=M^{2} M_{y y}+M M_{y}^{2}
\end{aligned}
$$

and, from Equation (23), it is possible to write

$$
\begin{aligned}
x_{y} & =\frac{1}{M} \\
x_{T} & =M M_{y y}+M_{y}^{2}-3 M
\end{aligned}
$$

whose compatibility condition $x_{y T}=x_{T y}$ yields

$$
\left[\frac{1}{M}\right]_{T}=\left[M M_{y y}+M_{y}^{2}-3 M\right]_{y}
$$

or, in a more compact form,

$$
[M]_{T}+\left[M^{3}\left(M_{y y}-1\right)\right]_{y}=0
$$

We shall denote Equation (27) as RKdV due to the fact that it is the reciprocal version of the $\mathrm{KdV}$ equation.

Reciprocal Transformations for the Spectral Problem of KdV

Let us write again the Lax pair (19) for KdV

$$
\begin{aligned}
& \psi_{x x}=(m-\lambda) \psi \\
& \psi_{t}=(2 m+4 \lambda) \psi_{x}-m_{x} \psi
\end{aligned}
$$

where the subscripts have been removed for simplicity. The eigenfunction in the new variables could be introduced as

$$
\psi(x, t)=\Phi(y, T)
$$

By using (24) and (25) in (28), we get the Lax pair for the reciprocal version (27) of KdV. This Lax pair reads

$$
\begin{aligned}
\Phi_{y y} & =-\frac{M_{y}}{M} \Phi_{y}+\left[\frac{1}{M}-\frac{\lambda}{M^{2}}\right] \Phi \\
\Phi_{T} & =\left(M^{2} M_{y y}+M M_{y}^{2}-M^{2}+4 \lambda M\right) \Phi_{y}-M M_{y} \Phi
\end{aligned}
$$

Notice that the Painlevé test cannot be applied to (27). Nevertheless, the corresponding reciprocal transformation to an equation with the Painlevé property, such as (17), yields the appropriate Lax pair.

\section{Reciprocal-Miura Transformations between RKdV and RmKdV}

As we said before, Equations (1) and (17) are related through the Miura transformation [11,12],

$$
m=u^{2}+k u_{x}, \quad k= \pm 1
$$

The reciprocal transformations introduced in the previous sections imply that

$$
u(x, t)=U(z, \tau), \quad m(x, t)=M(y, T)
$$


where the transformations among the independent variables are

$$
\begin{aligned}
& d x=\frac{d z}{u}-\frac{2 u^{3}-u_{x x}}{u} d \tau \\
& d x=\frac{d y}{m}-\frac{3 m^{2}-m_{x x}}{m} d T \\
& t=\tau=T
\end{aligned}
$$

A question immediately arises, is there any sort of Miura relation between $M(y, T)$ and $U(z, \tau)$ ?. Let us proceed to answer this question. By using (11) and (32) in (31), we have

$$
M=U\left(U+k U_{z}\right)
$$

Equation (34) can be considered to be the reciprocal version of the Miura map (31). Nevertheless, it should be combined with the transformation between the set of variables $\{z, \tau\}$ and $\{y, T\}$, defined in (33a) and (33b). The combination of these transformations gives rise to

$$
\begin{aligned}
& d y=\frac{m}{u} d z+\left[3 m^{2}-m_{x x}+\frac{m}{u}\left(u_{x x}-2 u^{3}\right)\right] d \tau \\
& T=\tau
\end{aligned}
$$

This result allows us to write a Miura-reciprocal transformation between RmKdV (13) and $\mathrm{RKdV}(27)$ as

$$
\begin{aligned}
& M(y, T)=U(z, \tau)\left(U(z, \tau)+k U_{z}(z, \tau)\right) \\
& d y=\left[U+k U_{z}\right] d z+\left[\left(U^{4}-U^{3} U_{z z}\right)+k\left(U^{4}-U^{3} U_{z z}\right)_{z}\right] d \tau \\
& T=\tau
\end{aligned}
$$

The whole picture concerning the relations among the integrable equations and their associated spectral problems analyzed in this article can be illustrated in Figure 1.

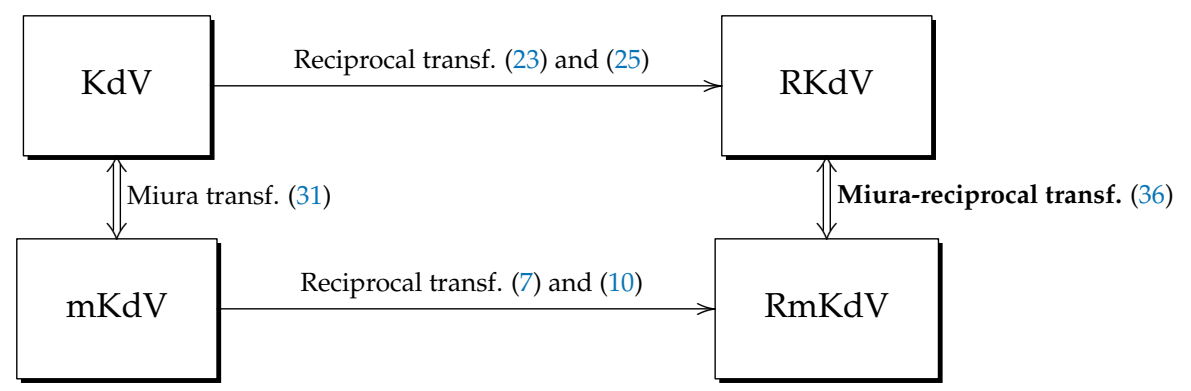

Figure 1. Miura-reciprocal transformation for RKdV and RmKdV.

The results obtained in the previous sections may be summarized in the following theorem and corollary:

Theorem 1. Let $u(x, t)$ be a solution of the $m K d V$ equation (1) and $m(x, t)$ be a solution of the $K d V$ equation (17), related by the Miura map (31). The conservative form of $K d V$ and $m K d V$ allows the introduction of the reciprocal transformations

$$
\begin{array}{lll}
d x=\frac{d z}{u}-\frac{2 u^{3}-u_{x x}}{u} d \tau, & t=\tau, & u(x, t)=U(z, \tau) \\
d x=\frac{d y}{m}-\frac{3 m^{2}-m_{x x}}{m} d T, & t=T, & m(x, t)=M(y, T)
\end{array}
$$


such that (37), when applied to $m K d V$, provides the reciprocal $m K d V$ (RmKdV) equation

$$
U_{\tau}+\left[U^{3}\left(U_{z z}-U\right)\right]_{z}=0
$$

whilst (38) gives rise to the reciprocal version of $K d V(R K d V)$,

$$
[M]_{T}+\left[M^{3}\left(M_{y y}-1\right)\right]_{y}=0
$$

Then, the Miura-reciprocal transformation defined by

$$
\begin{aligned}
& M(y, T)=U(z, \tau)\left(U(z, \tau)+k U_{z}(z, \tau)\right) \\
& d y=\left[U+k U_{z}\right] d z+\left[\left(U^{4}-U^{3} U_{z z}\right)+k\left(U^{4}-U^{3} U_{z z}\right) z\right] d \tau, \quad k= \pm 1 \\
& T=\tau
\end{aligned}
$$

establishes a map between RmKdV (39) and RKdV (40).

Corollary 1. Reciprocal transformations (37) and (38) induce a transformation over the spectral problems of $m K d V(2)$ and $K d V(28)$, respectively. Then, the resulting Lax pairs for RmKdV (15) and RKdV (30) are linked via the map (41) such that each component of the eigenvector $\vec{\Psi}(z, \tau)=\left(\Psi_{1}, \Psi_{2}\right)^{\top}$ in (15) satisfies the scalar linear problem for $\Phi(y, T)$ in (30).

\section{Lie Symmetries for the Transformed Spectral Problems}

As the final section of this paper, we identify the Lie point symmetries [23-25] for the spectral problems given in (15) and (30). Lie symmetries were first introduced by Lie [25] in order to solve ordinary differential equations (ODEs) or reduce a system of equations to a simpler form [26,27]. Lie point symmetries represent a powerful tool, although they involve lengthy calculations for the most part. Each Lie point symmetry leads us to a reduced version of the equation with the number of independent variables diminished by one. This fact implies that any solution of a PDE can be derived by its simmilarity reduction to an ODE. Much less frequent is the identification of Lie symmetries of the spectral problem of a PDE system [28]. The introduction of an spectral (or non-isospectral) parameter in a linear problem without spectral parameter through one-parameter groups of Lie point symmetries was proposed by Levi et al. [18,29]. This group interpretation of the spectral parameter has been extensively studied by Cieśliński et al. in several papers $[19,30,31]$ and by Marvan et al. [20,32,33]. Lie symmetries for the Lax pair [21] of a PDE provide much more information than just the symmetries of the PDE itself since they allow to get the similarity reductions of both the PDE and the spectral problem [34]. This approach requires to consider the spectral parameter as an independent variable and the eigenfunctions as fields $[35,36]$.

\subsection{Lie Point Symmetries for RKdV}

Let us consider a one-parameter Lie group [26] of infinitesimal transformations of the independent variables $\{y, T\}$, one dependent field $M(y, T)$, the spectral parameter $\lambda$ and the eigenfunction $\Psi(y, T, \lambda)$, given by

$$
\begin{aligned}
\tilde{y} & =y+\epsilon \xi_{y}(y, t, M, \lambda, \Phi) \\
\tilde{T} & =T+\epsilon \tilde{\xi}_{T}(y, t, M, \lambda, \Phi) \\
\tilde{\lambda} & =\lambda+\epsilon \xi_{\lambda}(y, t, M, \lambda, \Phi) \\
\tilde{M} & =M+\epsilon \eta_{M}(y, t, M, \lambda, \Phi) \\
\tilde{\Phi} & =\Psi+\epsilon \eta_{\Phi}(y, t, M, \lambda, \Phi)
\end{aligned}
$$


where $\epsilon$ is the group parameter. The associated vector field that generates these infinitesimal transformations reads

$$
X_{\mathrm{RKdV}}=\xi_{y} \frac{\partial}{\partial y}+\xi_{T} \frac{\partial}{\partial T}+\xi_{\lambda} \frac{\partial}{\partial \lambda}+\eta_{M} \frac{\partial}{\partial M}+\eta_{\Phi} \frac{\partial}{\partial \Phi}
$$

This infinitesimal transformation induces a well known one in the derivatives of the fields and it must preserve the invariance of the starting system of PDEs. By applying Lie's method [23-25], this procedure yields an overdetermined system of PDEs for the infinitesimals called the determining equations, whose solutions provide the classical symmetries. We have computed these symmetries with the help of Maple for the Lax pair given in (30). The result is

$$
\begin{aligned}
\xi_{y} & =C_{1} y+C_{2} \\
\xi_{T} & =-3 C_{1} T+C_{3} \\
\eta_{M} & =2 C_{1} M \\
\xi_{\lambda} & =2 C_{1} \lambda \\
\eta_{\Phi} & =f(\lambda) \Phi
\end{aligned}
$$

where $C_{i}, i=1 \ldots 3$ are arbitrary constants. $f(\lambda)$ is an arbitrary function of $\lambda$ which is a consequence of the invariance of the Lax pair (30) when $\Phi$ is multiplied by an arbitrary function of $\lambda$. Symmetries (44) yield the three-dimensional Lie algebra spanned by $\left\langle y \partial_{y}-3 T \partial_{T}+2 \lambda \partial_{\lambda}+2 M \partial_{M}, \partial_{y}, \partial_{T}\right\rangle$ and the infinite-dimensional subalgebra $\left\langle f(\lambda) \partial_{\Phi}\right\rangle$ due to the presence of the arbitrary function $f(\lambda)[24,27]$. For each parameter we can have a similarity reduction. We shall identify the reduction associated to $C_{1}$ because the symmetries connected to $C_{2}$ and $C_{3}$ are no more than translations which yield trivial reductions.

Reduction Associated to $C_{1}$

The vector field associated to $C_{1}$ is

$$
X_{1}=y \frac{\partial}{\partial y}-3 T \frac{\partial}{\partial T}+2 \lambda \frac{\partial}{\partial \lambda}+2 M \frac{\partial}{\partial M}
$$

and the reduction can be obtained through the integration of the characteristic system

$$
\frac{d y}{y}=\frac{d T}{-3 T}=\frac{d \lambda}{2 \lambda}=\frac{d M}{2 M}=\frac{d \Phi}{0}
$$

This integration yields

- $\quad$ Reduced independent variable

$$
Y=y T^{1 / 3}
$$

- $\quad$ Reduced field

$$
N(Y)=M(y, T) T^{2 / 3}
$$

- $\quad$ Reduced spectral parameter

$$
\beta=\lambda T^{2 / 3}
$$

where $\beta$ is the new spectral parameter.

- $\quad$ Reduced eigenfunction

$$
\bar{\Phi}(Y, \beta)=\Phi(y, T)
$$

- Reduced Lax pair

$$
\begin{aligned}
& \bar{\Phi}_{Y Y}=-\frac{N_{Y}}{N} \bar{\Phi}_{Y}+\frac{N-\beta}{N^{2}} \bar{\Phi} \\
& \beta \bar{\Phi}_{\beta}=\left[\frac{3}{2}\left(N^{2} N_{Y Y}+N N_{Y}^{2}-N^{2}\right)+6 \beta N-\frac{Y}{2}\right] \bar{\Phi}_{Y}-\frac{3}{2} N N_{Y} \bar{\Phi}_{Y}
\end{aligned}
$$




\section{- $\quad$ Reduced ODE}

$$
\left[N^{3}\left(N_{Y Y}-1\right)\right]_{Y}+\frac{Y}{3} N_{Y}-\frac{2 N}{3}=0
$$

We should remark that this is an elegant way of obtaining a rather non-trivial Lax pair for a non-autonomous ODE. The introduction of the spectral parameter $\beta$ arises in a natural form.

\subsection{Lie Point Symmetries for $R m K d V$}

Let us consider now the spectral problem obtained in (15). In this case, we should consider Lie infinitesimal transformations for the independent variables $\{z, \tau\}$, one dependent field $U(z, \tau)$, the spectral parameter $\Lambda$ and the eigenfunctions $\vec{\Psi}(z, \tau, \Lambda)=\left(\Psi_{1}, \Psi_{2}\right)^{\top}$, given by

$$
\begin{aligned}
\tilde{z} & =z+\epsilon \xi_{z}\left(z, \tau, U, \Lambda, \Psi_{1}, \Psi_{2}\right) \\
\tilde{\tau} & =\tau+\epsilon \xi_{\tau}\left(z, \tau, U, \Lambda, \Psi_{1}, \Psi_{2}\right) \\
\tilde{\Lambda} & =\Lambda+\epsilon \xi_{\Lambda}\left(z, \tau, U, \Lambda, \Psi_{1}, \Psi_{2}\right) \\
\tilde{U} & =U+\epsilon \eta_{U}\left(z, \tau, U, \Lambda, \Psi_{1}, \Psi_{2}\right) \\
\tilde{\Psi_{1}} & =\Psi_{1}+\epsilon \eta_{\Psi_{1}}\left(z, \tau, U, \Lambda, \Psi_{1}, \Psi_{2}\right) \\
\tilde{\Psi}_{2} & =\Psi_{2}+\epsilon \eta_{\Psi_{2}}\left(z, \tau, U, \Lambda, \Psi_{1}, \Psi_{2}\right)
\end{aligned}
$$

Lie symmetries have been computed using Maple, resulting in

$$
\begin{aligned}
\xi_{z} & =D_{2} \\
\xi_{\tau} & =3 D_{1} \tau+D_{3} \\
\eta_{U} & =-D_{1} U \\
\xi_{\Lambda} & =-D_{1} \Lambda \\
\eta_{\Psi_{1}} & =g(\Lambda) \Psi_{1} \\
\eta_{\Psi_{2}} & =g(\Lambda) \Psi_{2}
\end{aligned}
$$

The most interesting reduction arises from the one associated to $D_{1}$, since parameters $D_{2}, D_{3}$ provide trivial reductions.

Reductions Associated to $D_{1}$

The characteristic system for the symmetry related to $D_{1}$ is

$$
\frac{d z}{0}=\frac{d \tau}{3 \tau}=\frac{d \Lambda}{-\Lambda}=\frac{d U}{-U}=\frac{d \Psi_{1}}{0}=\frac{d \Psi_{2}}{0}
$$

whose integration yields the following reduction

- $\quad$ Reduced independent variable

$$
Z=z
$$

- $\quad$ Reduced field

$$
H(Z)=U(z, \tau) \tau^{1 / 3}
$$

- $\quad$ Reduced spectral parameter

$$
\alpha=\Lambda \tau^{1 / 3}
$$

where $\alpha$ is the new spectral parameter for the reduced spectral problem.

- $\quad$ Reduced eigenfunctions

$$
\begin{aligned}
& \Omega_{1}(Z, \alpha)=\Psi_{1}(z, \tau) \\
& \Omega_{2}(Z, \alpha)=\Psi_{2}(z, \tau)
\end{aligned}
$$

denoted in the following as $\vec{\Omega}=\left(\Omega_{1}, \Omega_{2}\right)^{\top}$. 
- $\quad$ Reduced Lax pair

$$
\begin{aligned}
& \vec{\Omega}_{Z}=\hat{B} \vec{\Omega} \\
& \vec{\Omega}_{\alpha}=3 \hat{C} \vec{\Omega}
\end{aligned}
$$

where $\hat{B}$ and $\hat{C}$ are the $2 \times 2$ matrices

$$
\begin{aligned}
& \hat{B}=\left(\begin{array}{cc}
-1 & \frac{\alpha}{H} \\
\frac{\alpha}{H} & 1
\end{array}\right) \\
& \hat{C}=\left(\begin{array}{cc}
4 \alpha H & H H_{Z Z}+H_{Z}^{2}-2 H H_{Z}-4 \alpha^{2} \\
H H_{Z Z}+H_{Z}^{2}+2 H H_{Z}-4 \alpha^{2} & -4 \alpha H
\end{array}\right)
\end{aligned}
$$

- $\quad$ Reduced ODE

$$
\left[H^{3}\left(H_{Z Z}-H\right)\right]_{Z}-\frac{H}{3}=0
$$

As in the case of RKdV, the spectral problem of the reduced ODE is nicely obtained through the introduction of a spectral parameter $\alpha$ which arises from the reduction of the spectral parameter $\Lambda$ of the former Lax pair.

\section{Conclusions}

In this paper we have presented reciprocal transformations for the spectral problems of $\mathrm{KdV}$ and $\mathrm{mKdV}$. The link between the resulting reciprocal equations is established by means of the so-called Miura-reciprocal transformation, which stands for a combination of the proposed reciprocal transformations and the Miura map inherited from the existing relation between $\mathrm{KdV}$ and $\mathrm{mKdV}$. These results are compiled in Theorem 1 and Corollary 1.

Furthermore, the classical Lie symmetries for the reciprocal version of these spectral problems are identified. These symmetries may straightforwardly yield non-trivial similarity reductions. When dealing with the Lax pair instead of just the equation itself, we also get the reduced spectral problem for the reduced ODE. The reduction of the spectral parameter plays an important role in this process.

Author Contributions: These authors contributed equally to this work. All authors have read and agreed to the published version of the manuscript.

Funding: This research has been supported in part by MICINN (Grant PID2019-106820RBC22) and Junta de Castilla y León (Grant SA256P18). P. Albares also acknowledges support from the predoctoral grant FPU17/03246.

Institutional Review Board Statement: Not applicable.

Informed Consent Statement: Not applicable.

Data Availability Statement: Not applicable.

Conflicts of Interest: The authors declare no conflict of interest.

\section{References}

1. Clarkson, P.A.; Fokas, A.S.; Ablowitz, M.J. Hodograph transformations of linearizable partial differential equations. SIAM J. Appl. Math. 1989, 49, 1188-1209. [CrossRef]

2. Rogers, C.; Shadwick, W.F. Bäcklund Transformations and Their Applications; Mathematics in Science and Engineering; Academic Press: New York, NY, USA,1982; Volume 161.

3. Rogers, C. Reciprocal transformations in (2+1) dimensions. J. Phys. A Math. Gen. 1986, 19, L491-L496. [CrossRef]

4. Rogers, C. The Harry Dym equation in $2+1$ dimensions: A reciprocal link with the Kadomtsev-Petviashvili equation. Phys. Lett. A 1987, 120, 15-18. [CrossRef]

5. Estévez, P.G. Reciprocal transformations for a spectral problem in $2+1$ dimensions. Theor. Math. Phys. 2009, 159, 763-769. [CrossRef] 
6. Estévez, P.G. Generalized Qiao hierarchy in $2+1$ dimensions: Reciprocal transformations, spectral problem and non-isospectrality. Phys. Lett. A 2011, 375, 537-540. [CrossRef]

7. Hone, A.N.W. Reciprocal link for $2+1$-dimensional extensions of shallow water equations. Appl. Math. Lett. 2000, 13, 37-42. [CrossRef]

8. Estévez, P.G.; Prada, J. Hodograph Transformations for a Camassa-Holm hierarchy in $2+1$ dimensions. J. Phys. A Math. Gen. 2005, 38, 1287-1297. [CrossRef]

9. Estévez, P.G.; Sardón, C. Miura reciprocal transformations for hierarchies in $2+1$ dimensions. J. Nonlin. Math. Phys. 2013, 20, 552-564. [CrossRef]

10. Degasperis, A.; Holm, D.D.; Hone, A.N. A new integral equation with peakon solutions. Theor. Math. Phys. 2002, 133, 1463-1474. [CrossRef]

11. Ablowitz, M.J.; Kruskal, M.; Segur, H. A note on Miura's transformation. J. Math. Phys. 1979, 20, 999-1003. [CrossRef]

12. Sakovich, S.Y. On Miura transformations of evolution equations. J. Phys. A Math. Gen. 1993, 26, L369-L373. [CrossRef]

13. Weiss, J. The Painlevé property for partial differential equations. II: Bäcklund transformation, Lax pairs, and the Schwarzian derivative. J. Math. Phys.1983, 24, 1405-1413. [CrossRef]

14. Sakovich, A.; Sakovich, S. The Short Pulse Equation Is Integrable. J. Phys. Soc. Jpn. 2005, 74, 239-241. [CrossRef]

15. Sakovich, S. Transformation and integrability of a generalized short pulse equation. Commun. Nonlinear Sci. Numer. Simul. 2016, 39, 21-28. [CrossRef]

16. Sakovich, S. Integrability of a Generalized Short Pulse Equation Revisited. Res. Appl. Math. 2018, 2, 1-11. [CrossRef]

17. Ablowitz, M.J.; Clarkson, P.A. Solitons, Nonlinear Evolution Equations and Inverse Scattering; London Mathematical Society, Lecture Notes Series 149; Cambridge University Press: Cambridge, UK, 1991.

18. Levi, D.; Sym, A. Integrable systems describing surfaces of non-constant curvature. Phys. Lett. A 1990, 149, 381-387. [CrossRef]

19. Cieslinski, J. Group interpretation of the spectral parameter in the case of nonhomogeneous, nonlinear Schrödinger system. J. Math. Phys. B 1993, 34, 2372-2384. [CrossRef]

20. Marvan, M. On the Spectral Parameter Problem. Acta Appl. Math. 2010, 1096, 239-255. [CrossRef]

21. Estévez, P.G.; Gandarias, M.L.; Prada, J. Symmetry reductions of a $2+1$ Lax pair. Phys. Lett. A 2005, 343, 40-47. [CrossRef]

22. Camassa, R.; Holm, D.D. An integrable shallow water equation with peaked solitons. Phys. Rev. Lett. 1993, 71, 1661-1664. [CrossRef]

23. Bluman, G.W.; Kumei, S. Symmetries and Differential Equations; Springer: New York, NY, USA, 1989.

24. Olver, P.J. Applications of Lie Groups to Differential Equations; Springer: New York, NY, USA, 1993.

25. Lie, S. Theorie der Transformationgruppen; B. G.Teubner: Leipzig, Germany, 1888.

26. Stephani, H. Differential Equations. Their Solutions Using Symmetries; Callum, M.M., Ed.; Cambridge University Press: Cambridge, UK, 1989.

27. Snobl, L.; Winternitz, P. Classification and Identification of Lie Algebras; CRM Monograph Series, American Mathematical Society: Providence, RI, USA, 2014.

28. Legare, M. Symmetry reductions of the Lax pair of the four-dimensional Euclidean self-dual Yang-Mills equations. J. Nonlinear Math. Phys. 1996, 3, 266-285. [CrossRef]

29. Levi, D.; Sym, A.; Tu, G.Z. A Working Algorithm to Isolate Integrable Surfaces in $E^{3}$, Preprint n. 761; Dipartimento di Fisica, Università di Roma: Roma, Italy, October 1990.

30. Cieslinski, J. Nonlocal symmetries and a working algorithm to isolate integrable geometries. J. Phys. A Math. Gen. 1993, 26, L267-L271. [CrossRef]

31. Cieslinski, J.; Goldstein, P.; Sym, A. On integrability of the inhomogeneous Heisenberg ferromagnet model: examination of a new test. J. Phys. A Math. Gen. 1994, 27, 1645-1664. [CrossRef]

32. Krasil'shchik, J.; Marvan, M. Coverings and Integrability of the Gauss-Mainardi-Codazzi Equation. Acta Appl. Math. 1999, 56, 217-230. [CrossRef]

33. Marvan, M. On the Horizontal Gauge Cohomology and Nonremovability of the Spectral Parameter. Acta Appl. Math. 2002, 72, 51-65. [CrossRef]

34. Bruzón, M.S.; Estévez, P.G.; Gandarias, M.L.; Prada, J. $1+1$ spectral problems arising from the Manakov-Santini system. J. Phys. A Math. Theor. 2010, 43, 495204. [CrossRef]

35. Albares, P.; Conde, J.M.; Estévez, P.G. Spectral problem for a two-component nonlinear Schrödinger equation in $2+1$ dimensions: Singular manifold method and Lie point symmetries. Appl. Math. Comput. 2019, 355, 585-594. [CrossRef]

36. Albares, P.; Estévez, P.G.; Lejarreta, J.D. Derivative non-linear Schrödinger equation: Singular manifold method and Lie symmetries. Appl. Math. Comput. 2021, 400, 126089. 